\title{
Lumen
}

Selected Proceedings from the Canadian Society for Eighteenth-Century Studies

\section{Entre brevitas et digressions narratives : le dilemme du romancier dans la première moitié du XVIII ${ }^{\mathrm{e}}$ siècle}

\section{Stéphanie Bouabane}

Volume 18, 1999

Representations of Time in the XVIIIth Century

Le temps et ses représentations au dix-huitième siècle

URI : https://id.erudit.org/iderudit/1012364ar

DOI : https://doi.org/10.7202/1012364ar

Aller au sommaire du numéro

Éditeur(s)

Canadian Society for Eighteenth-Century Studies / Société canadienne d'étude du dix-huitième siècle

ISSN

1209-3696 (imprimé)

1927-8284 (numérique)

Découvrir la revue

Citer cet article

Bouabane, S. (1999). Entre brevitas et digressions narratives : le dilemme du romancier dans la première moitié du XVIII ${ }^{\mathrm{e}}$ siècle. Lumen, 18, 13-25.

https://doi.org/10.7202/1012364ar

Copyright (c) Canadian Society for Eighteenth-Century Studies / Sociéte canadienne d'étude du dix-huitième siècle, 1999
Ce document est protégé par la loi sur le droit d'auteur. L'utilisation des services d'Érudit (y compris la reproduction) est assujettie à sa politique d'utilisation que vous pouvez consulter en ligne.

https://apropos.erudit.org/fr/usagers/politique-dutilisation/ 


\section{Entre brevitas et digressions narratives: le dilemme du romancier dans la première moitié du XVIIIe siècle}

'Un auteur ne peut s'abstenir d'une préface: celle-ci sera courte; elle est finie. ${ }^{1} \mathrm{Si}$ Godart de Beauchamps élude de manière aussi rapide la nécessité de se plier à la 'convention préfacielle', la plupart des romanciers au XVIIIe siècle pratique également une dérision non moins ostentatoire qu'ils développent plutôt longuement. Tous les traités de rhétorique s'accordent pour reconnaître la nécessité de faire précéder le discours - judiciaire ou littéraire - d'un texte présentatif. C'est ainsi que dans la pure tradition de Cervantes, les romanciers s'emploient obligeamment à décrire les difficultés qui ont retardé la rédaction de leur préface, à l'exemple de Marivaux dans La voiture embourbée:

Dieu soit béni, me voilà délivré d'un grand fardeau, et je ris encore du personnage que j'allais faire, si j'avais été obligé de soutenir ma préface. Adieu, j'aime mieux mille fois couper court, que d'ennuyer par trop de longueur. Passons à l'ouvrage. $^{2}$

Des gnômai soulignent cette prise de conscience d'une rhétorique usée, caractérisée par l'ennui que le discours d'ouverture ne peut manquer de susciter. On connaît la célèbre préface des Lettres persanes, où l'auteur, qui se refuse à rédiger 'une chose très ennuyeuse, placée dans un lieu déjà très ennuyeux de lui-même', ${ }^{3}$ se défend à juste titre de donner une trop longue préface. Il convient de ne pas fatiguer le lecteur, 'défaut que l'on ne pardonne point à l'auteur d'un roman ou d'une nouvelle. ${ }^{4} \mathrm{La}$ préface est assimilée à son modèle oratoire, l'exorde, présenté comme un 'mal nécessaire': 'quelques rieurs [ont] dit que les exordes du discours sont comme les orgues des églises, qui ne servent qu'à allonger le service. $^{, 5}$

Cette présence obligatoire se justifie ensuite par l'exposition du but de la préface, qui répond à la fonction de captatio benevolentio de l'exorde, défini comme 'un développement qui prépare comme il faut l'esprit de $l^{\prime}$ auditeur à suivre le reste du discours. ${ }^{6}$ Comme l'exorde, la préface doit s'efforcer de rendre le lecteur 'bienveillant, attentif, apte à comprendre." 
Nombre de lieux communs traduisent l'autodérision exprimée par les romanciers à l'égard de ce discours imposé, dont la nécessité fait paradoxalement - l'objet d'un consensus: ' $[\mathrm{m}]$ on livre aura une préface, ne fût-elle que de deux lignes; pourrais-je paraître dans le monde avec quelque honneur, sans être annoncé par une préface? ${ }^{8}$

\section{La brièveté préfacielle: de l'utilité de la prétérition}

Le romancier se trouve confronté à un dilemme: mis en demeure de rédiger une préface mais conscient de l'ennui de sa démarche, il lui faut satisfaire exigences stylistiques et goût du lectorat: '[u]n excès de longueur dans une préface serait un défaut, comme c'en est un d'affecter ridiculement de commencer un ouvrage sans préface et sans introduction. ${ }^{\prime 9} C^{\prime}$ est ainsi qu'il n'est guère 'permis d'allonger une préface comme on tire un lingot $\mathrm{d}^{\prime}$ or $^{\prime},{ }^{10}$ surtout si celle-ci se révèle hors de propos: ' $[\mathrm{u}]$ ne longue préface serait ici hors d'œuvre. Il suffit de dire en peu de mots quelle est la fin qu'on a eue en écrivant cette histoire, et l'ordre qu'on y a gardé. ${ }^{11}$

Comment remplir 1"obligation préfacielle' sans pour autant ennuyer le lecteur? La brièveté s'avère être la seule issue. Qualité majeure du discours d'ouverture, elle est ainsi plébiscitée par les romanciers qui la considèrent, à la suite d'Hermogène, comme partie prenante - avec la clarté et la crédibilité - des virtutes narrationis. ${ }^{12}$ Enclins à reproduire l'idéal de brièveté qui contribue à conférer puissance et vivacité au discours, les romanciers n'hésitent pas à utiliser à cet effet la technique de la pointe, la sentence brève et brillante dont les qualités de style sont nouveauté, vigueur, brièveté et variété. ${ }^{13}$ Afin de se conformer au schéma qu'ils plébiscitent, les romanciers recourent également au trait rhétorique de la prétérition, procédé commode lorsque l'inspiration est censée faire défaut - topos romanesque s'il en est: '[q]ue j'aurais une vaste carrière à remplir, si je décrivais toutes [les vertus] dont vous êtes pourvu!' ${ }^{14}$ Plutôt que d'effectuer un portrait en bonne et due forme, l'auteur souligne la faiblesse de sa plume au regard des qualités de son dédicataire. La prétérition est à rapprocher de l'excusatio propter infirmitatem, pendant négatif de l'amplificatio qui, en affirmant l'incapacité de l'orateur à traiter son sujet, exagère l'importance de celui-ci. ${ }^{15}$ Cette formule topologique a pour fonction de s'attirer les suffrages du public et s'inscrit dans l'entreprise de séduction de la captatio benevolentio:

Je ne l'emploierai point à justifier les défauts qui peuvent se rencontrer dans mon ouvrage, et à prouver au public qu'il aurait tort de s'ennuyer à sa lecture; je ne la fais que pour avertir le lecteur qu'il me doit être fort obligé: car au moins, si je l'ennuie, ne le ferai-je pas longtemps. ${ }^{16}$ 
Ce trait rhétorique, qui consiste à 'feindre de ne pas vouloir dire ce que néanmoins on dit très clairement, et souvent même avec force ${ }^{17}$ se présente ici comme une dérobade. Le romancier écrit une préface 'en expliquant qu' [il] ne le fera pas, ou en évoquant toutes celles qu'[il] aurait pu faire': ${ }^{18}$ ' $[\mathrm{s}]^{\prime}$ il était à propos d'enfler cette préface, l'on déploierait ici un catalogue qui sans doute embarrasserait bien du monde. ${ }^{19}$ Une sorte de 'coquetterie littéraire' ou de 'précaution oratoire ${ }^{20}$ semble présider au jugement porté par le romancier sur le genre préfaciel. La prétérition permet de renseigner le lecteur sur la fonction conventionnelle de toute préface et de la subvertir dans le même temps; ainsi, nul besoin de s'appesantir dans un discours dit 'de captation': '[j]e ne préviendrai point le lecteur sur le volume que je présente aujourd'hui; les longues préfaces ne conviennent qu'aux ouvrages d'érudition, et les miens ne sont pas de ce genre [...]. ${ }^{21}$

La préface romanesque du XVIIIe siècle a relation directe avec la dimension autoréflexive des discours d'ouverture tels que l'entendaient les auteurs des siècles précédents; il suffit de l'étudier au regard de textes comme le 'Prologue' du Gargantua. Ces passages métatextuels, axés sur la forme même qui les soutient, se multiplient cependant et tendent à investir non seulement la préface, mais plus généralement toutes les formes de discours préliminaires - comme l'avertissement au lecteur ou l'épître dédicatoire.

\section{La brièveté diégétique: un idéal stylistique?}

Si le romancier estime veiller dans sa préface à ne pas provoquer l'ennui du lecteur, il déclare de même ne point vouloir faire languir sa narration: '[l]es plus courtes folies sont toujours les meilleures, ainsi, mon cher lecteur, n'attendez de ma plume ni une longue histoire, ni une longue préface. Le sort des ouvrages diffus est d'ennuyer, et ces sortes d'ouvrages ne le sont toujours que trop. ${ }^{22}$

La question de la brièveté peut par conséquent être envisagée en rapport avec tout ce qui constitue le texte, que les revendications esthétiques de l'auteur soient exposées dans le paratexte ou dans la diégèse. Le discours $\mathrm{d}$ 'introduction aborde $\mathrm{d}$ 'ailleurs très fréquemment les questions relatives à la genèse du manuscrit. A cet effet, il est courant que l'auteur attribue son texte à autrui, dans la perspective classique de la théorie imitative: il omet rarement, en ce cas, de signaler les modifications qu'il a fait subir au modèle dont il se réclame, qu'il s'agisse d'une adaptation ou d'une simple traduction. Le texte-source est présenté de façon systématique comme une narration interminable qu'il était nécessaire d'élaguer. Le pseudo-éditeur de La Rosalinde dit avoir 'retranché un 
tiers au moins de son original': '[d]e dix livres italiens, je n'en ai fait que huit français et il n'a pas tenu à moi de devoir cette brièveté autant à la précision du style qu'aux suppressions que j'ai faites. ${ }^{23}$ L'éditeur parle ensuite $d^{\prime \prime}$ une foule de descriptions entassées les unes sur les autres [...] que tout le monde sait par cœur', ${ }^{24}$ de 'dissertations énormes ${ }^{25}$ qui 'interrompent le fil de l'histoire et dépitent avec raison le lecteur intéressé. ${ }^{26}$ Les défauts stylistiques de l'original justifient toujours les remaniements: '[n]arrations confuses et embarrassées, événements obscurs ou mal développés, faits importants noyés dans un déluge de réflexions inutiles, répétitions ennuyeuses, détails puérils, confusion assommante de noms barbares[....$^{27}$ Le pseudo-traducteur de Guzman $d^{\prime}$ Alfarache reproche lui aussi à son prédécesseur d'avoir interrompu sans arrêt le fil du récit ' pour se jeter dans de longues déclamations contre les mœurs. ${ }^{28}$ Les lecteurs abandonnent sans regret babillards et auteurs de sermons dont le bavardage rompt avec le précepte horatien. Pour mêler l'utile à l'agréable, il convient en effet de cacher les vérités sous le voile d'une fiction plaisante, afin de laisser au lecteur le soin de les découvrir:

[q]ue vos discours instructifs soient courts, autrement on ne les retiendra point. [...] Tout ce qu'il y a de trop s'écoule. C'est autant de bien perdu. Au lieu qu'une instruction laconique, ne faisant que donner matière à réflexions, laisse aux lecteurs le secret plaisir de les faire. ${ }^{29}$

C'est ainsi que la concision n'implique en aucun cas un appauvrissement de la pensée: cette abundantia in brevitate, qui renvoie à l'héritage baroque de la pointe, de l'agudeza chères à Gracián, Tesauro ou Castiglione, se traduit à un niveau microstructural par la figure de l'enthymème. En supprimant une proposition suffisamment claire pour que l'imagination puisse la rétablir de façon logique, l'écrivain reproduit la rapidité de la pensée. Pour Arnauld et Nicole, 'une des principales beautés d'un discours est d'être plein de sens, et de donner occasion à l'esprit de former une pensée plus étendue que n'est l'expression. ${ }^{\prime 30}$ Le lecteur éprouve du plaisir à combler les vides: 'cette suppression flatte la vanité de ceux à qui on parle, en se remettant de quelque chose à leur intelligence $[. ..] .{ }^{31}$ Nul besoin, par conséquent, de se montrer dogmatique à outrance: il est parfois bien plus agréable de goûter les charmes de l'implicite. Les romanciers savent eux aussi que le lecteur 'est bien aise de coopérer, et d'avoir les honneurs de certaines découvertes. ${ }^{, 32}$

Le traducteur peut donc s'accorder une grande liberté de composition et d'écriture, pourvu qu'il garde à l'esprit l'intérêt du lecteur, toujours lié, à l'époque, à la poétique de la brevitas. Il ne doit pas hésiter à livrer une version très personnelle du texte, 'purgée des moralités superflues ${ }^{\prime 33}$ 
et des commentaires qui ralentissent l'action, que 'presque tous les lecteurs [...] sautent, pour suivre les aventures du héros. ${ }^{, 34}$ La traduction, dans la première moitié du siècle, se présente plutôt comme une réécriture au goût du jour et du pays de textes peu conformes aux habitudes des lecteurs. La préface fictive souligne les procédés d'adaptation du texte original et reproduit par là le principe de sélection qui est celui de toute littérature. L'écrivain se doit de trier, parmi les œuvres qui l'ont marqué, la matière susceptible d'être intégrée dans son ouvrage; c'est sur cette appropriation du texte que repose le principe de l'intertextualité. La brevitas est donc au premier chef le résultat d'une sélection.

Les romans-mémoires ou les romans épistolaires, dans la mesure où ils se présentent comme véridiques, jouent eux aussi sur la transformation d'un proto-texte fictif. Le préfacier se pose en éditeur de mémoires ou de lettres authentiques qu'il affirme avoir soigneusement triées: 'je ne vous en envoie que ce que j'ai cru digne d'être lu; et dans plus de cinq cents qui me sont tombées entre les mains, je n'en ai réservé que soixante-dix; [...] les amants s'écrivent souvent des choses qui ne peuvent intéresser qu'eux-mêmes. ${ }^{35}$

Les romanciers se montrent aussi soucieux de se conformer aux préceptes des anciens rhétoriciens, que de démarquer leurs ouvrages des romans du siècle précédent, 'volumes énormes remplis d'un fatras insoutenable et dont les derniers ne font que répéter $d^{\prime}$ une manière fort ennuyeuse les aventures que contiennent les premiers. ${ }^{\prime 36} \mathrm{~L}$ 'argument de la novitas leur permet de contourner ce travers: la nouveauté tient en l'occurrence dans la réduction quantitative effectuée par rapport aux romans précieux. Par dérision, les auteurs mettent en scène des personnages passionnés de lecture, jugeant 'le Cyrus trop court' et l'Astrée 'une brochure des plus minces. ${ }^{37} \mathrm{~L}$ 'on s'émerveille de la patience des lecteurs d'autrefois: '[c]'est une espèce de prodige que l'impatience naturelle de notre nation ait pu si longtemps s'accommoder $\mathrm{d}^{\prime}$ une lecture qui oblige à parcourir dix volumes avant que d'arriver au dénouement de l'intrigue. ${ }^{38}$ Est critiquée en particulier la multiplication des péripéties sentimentales et guerrières, qu'accompagne tout un arsenal de lieux communs (tromperies, passions, enlèvements, fausses morts, tribulations, que l'on trouvait dans le roman grec); ces traits constitutifs de l'esthétique baroque conduisent à l'éclatement du récit dans une succession d'histoires parallèles. ${ }^{39}$ Outre les complications qui alourdissent la narration et demandent au lecteur 'une application particulière pour les bien conduire, les démêler, et les rendre intelligibles', ${ }^{40}$ on tend à épargner au lecteur le caractère artificiel de réflexions morales qui s'écartent de l'intrigue et qu'il ne prend pas même la peine de lire: 
Je n'ai pas, à la vérité, imité ces auteurs qui grossissent leurs ouvrages par de longues réflexions, qu'ils augmentent encore en vous assurant qu'ils n'en feront plus. Je vous avouerai mon faible: dans ces sortes de livres, je passe ordinairement les monologues; et ainsi, j'ai cru qu'il valait mieux n'en point mettre, que de vous donner la peine de tourner six ou sept feuillets pour en trouver la fin. ${ }^{41}$

La longueur comme source d'ennui apparaît également dans des situations énonciatives qui miment une communication entre destinateur et récepteur. Il arrive fréquemment, dans la première moitié du siècle, que des personnages dialoguent à l'envi au sujet d'un ouvrage et que l'un des protagonistes reproche au conteur intradiégétique la longueur de son propos: 'on vous demande le récit de vos aventures particulières, que vous auriez dû conter très succinctement dans la conjoncture où nous sommes; et, au lieu de cela, vous nous venez conter celles d'une autre, avec des circonstances aussi frivoles qu'elles sont ennuyeuses. ${ }^{42}$ Ces allusions dans lesquelles l'auteur préfigure l'impatience du lecteur sont légion dans les trois premières décennies. Chez Hamilton, les personnages ont pour fonction d'abréger tout épisode annexe qui ne ferait pas avancer l'économie du récit et interrompent à dessein le narrateur afin de limiter les digressions: '[m]ais achève ton conte, car je commence à le trouver un peu long. ${ }^{, 43}$ L'auteur suppose connues les attentes du lecteur et les devance. Ici encore, cette technique passe par l'usage de la prétérition. Tout comme le romancier déclarait ne vouloir point écrire de préface, il dit ne point vouloir aborder tel ou tel sujet, l'un des topoi consistant à refuser de développer la généalogie du protagoniste. Prétextant comme il se doit l'intérêt du lecteur, il prétend vouloir mettre l'accent 'sur les faits intéressants dont [l'] histoire est remplie ${ }^{\prime 44}$ tout en délivrant une information objective et succincte:

Je n'entretiendrai point le lecteur des différents vents qui soufflèrent pendant le cours de notre navigation, du beau temps que nous eûmes, du mauvais que nous essuyâmes, des rencontres indifférentes que nous fîmes, ni des îles où nous fûmes obligés de mouiller pour faire eau, et renouveler nos vivres; ce détail ne serait ni intéressant, ni instructif, et mon dessein n'est pas d'ennuyer exprès le lecteur. ${ }^{45}$

Le lien entre promesses préfacielles et déclarations effectives de la diégèse est nécessairement étroit; bien plus, cette confrontation permet de mesurer la distance entre les deux types de discours et de montrer qu'ils relèvent le plus souvent de la pure convention rhétorique. 


\section{Eloge de la digression narrative: une exigence morale et stylistique}

L'ellipse descriptive que constitue l'usage de la prétérition peut notamment se justifier par le caractère indescriptible des scènes évoquées: '[j]e ne m'arrêterai point à décrire tous ses transports; $d$ 'ailleurs je réussirais mal, il est des situations qu' on ne saurait peindre. ${ }^{46}$ Par cette forme de superlatif implicite, l'auteur marque l'impuissance de toute expression et la supériorité des choses sur les mots: la distinction essentielle entre res et verba contribue à amplifier l'intensité de la scène. La négation pure et simple de la description, à l'image de la présentation négative, traduit la nature extraordinaire de l'objet. Ajoutons que la description détaillée (ekphrasis) largement actualisée dans le roman grec, est considérée par la critique comme un élément étranger au propos romanesque, dont les rhétoriciens du XVIIIe siècle conseillent de se défier. ${ }^{47}$ Partie prenante des progymnasmata, ${ }^{48} 1^{\prime}$ hypotypose est proscrite dans la narration dans la mesure où la concision contribue à la clarté du discours, au même titre que l'absence de défauts ou la compositio (c'est-à-dire l'arrangement des mots). Les romanciers ne reconnaissent pas à l'ekphrasis sa fonction d'illustration et d'embellissement du récit. Loin de privilégier une esthétique descriptive, ils mettent l'accent sur la diversification des intrigues. La brièveté s'applique ainsi aux microstructures aussi bien qu'à l'ensemble de la narration.

La prétérition - tout comme l'ellipse narrative, parfois simplement marquée par la mention d'un 'manuscrit effacé ${ }^{\prime 49}$ - signifie toutefois que la digression est potentiellement omniprésente dans le discours et exprime la liberté de l'écrivain. L'auteur, en mettant l'accent sur ce qu'il ne dit pas, suggère qu'il aurait pu s'étendre à l'infini - auctor pouvant signifier, étymologiquement, 'celui qui augmente.' Ces différentes 'esquives', qui offrent une représentation quantitative du texte, permettent ainsi d'appréhender celui-ci comme un objet matériel susceptible d'être modifié indéfiniment: '[p]oint de volume d'une grosseur plus énorme que celui que j'offrirais au public, si je voulais épuiser la matière que j'ai à traiter dans ce livre. ${ }^{50} \mathrm{Il}$ faut cependant se défier des déclarations d'usage. L'auteur condamne certes la pratique digressive dans sa préface, déclarant que s'il est 'assez malheureux pour [...] causer quelque ennui, ce ne sera pas du moins par la longueur du récit' ${ }^{51}$ mais tente tout de même d'introduire et de se faire pardonner ses excursus, soit au sein de la préface, soit au cours de la narration. Madame de Lintot reconnaît, après un récit intercalé de quarante-cinq pages, avoir perdu 'le fil de [sa] préface. ${ }^{.52}$ Enclin à commenter les événements ou à superposer plusieurs niveaux narratifs, un conteur intradiégétique évoque la prolixité de l'auteur: 
Pardonnez-moi ces petites digressions, mon cher marquis. Je les éviterai avec soin pour ne vous point ennuyer par un récit trop long. Ah! ne vous gênez point, repartit Mr d'Ozanne, on ne s'ennuie pas de vous entendre et ce que vous appelez digressions, sont des réflexions très naturelles dans la bouche d'un honnête homme. ${ }^{53}$

L'auditeur, chargé de réclamer la suite de l'histoire et de rétablir le lien avec le récit principal, relève explicitement la longueur du récit. Le lecteur, jugé par l'auteur bien indulgent de l'avoir écouté si longtemps ${ }^{54}$ et 'assez généreux pour excuser [ses] fréquentes digressions', ${ }^{55}$ voit sa patience honorée. Le narrateur, après avoir promis de ne point s'écarter du sujet, admet avoir allongé son récit plus que de raison en évoquant le 'faible qu' ont tous les hommes d'être trop longs sur ce qui les regarde, surtout si cela a quelque chose de flatteur. ${ }^{56} \mathrm{~L}$ 'excursus se présente ainsi comme l'une des preuves attestant la bonne foi du narrateur. Madame de Lussan et Marivaux font de ce prétexte un usage courant; dans le roman-mémoires, l'abondance verbale de la narratrice est constamment assimilée à la sincérité autobiographique: '... il faut qu'on s'accoutume de bonne heure à mes digressions. [J]e ne me gênerai point, je conterai toute ma vie, et si j'y mêle autre chose, c'est que cela se présentera sans que je le cherche. ${ }^{, 57}$ Des revendications d'ordre littéraire viennent se greffer à ces justifications inspirées des lieux communs narratifs. Elles sont une façon d'éviter l'uniformité, de plaire par 'la prodigieuse variété des matières ${ }^{58}$ et de 'délasser l'esprit': ${ }^{59}$ '[j]' avoue que je m'écarte à tout moment de mon sujet pour courir à droite et à gauche sur des terres étrangères, mais sans ces excursions, comment aurais-je pu me défendre d'une marche uniforme? ${ }^{60}$

Incapable 'de soutenir le même style ni le même caractère depuis la préface jusqu'au privilège', ${ }^{61}$ le romancier assimile l'abondance à la variété. Portraits, dialogues et autres digressions permettent d'introduire une part de raffinement et de complexité et de conférer au discours 'son charme, ses grâces persuasives': ${ }^{62}$

Un récit a plus d'agrément, quand il est animé de personnages divers et coupé de dialogues; on rend en outre plus vraisemblables les faits dont on parle, si l'on expose comment ils se sont passés; on les rend enfin plus faciles à comprendre, si l'on marque parfois des temps d'arrêt, au lieu de courir toujours avec une hâte déplacée. ${ }^{63}$

Si les théoriciens du roman conseillent d'éviter de 'plaquer' une moralité sur la narration - celle-ci se doit tirer naturellement des actions et des situations ${ }^{64}$ - la présence d'un contenu édifiant trouve sa justification. Le topos consiste à invoquer le respect des convenances ou encore 
la nécessité de défendre le roman de l'immoralisme dont on l'accuse. Le 'dilemme du roman' analysé par G. May ${ }^{65}$ est le prétexte même qui permet aux romanciers de développer certaines considérations morales, en rupture avec les contraintes stylistiques mentionnées auparavant. Cette tendance, qui s'accroît tout au long de la première moitié du siècle, se développe davantage à l'apparition du roman sensible des années soixante.

Fidèles en cela aux théoriciens du conceptisme, ${ }^{66}$ eux-mêmes influencés par les auteurs de la latinité d'argent - Sénèque, Pline et Tacite les romanciers, dans les premières décennies du XVIIIe siècle, apparentent le 'long parler' au 'trop parler.' Signifiant à l'exemple du rhétoricien Leven de Templery que 'la longueur rend ennuyeuses les choses même les plus agréables' ${ }^{67}$ ils revendiquent l'union idéale de la copia et de la brevitas. Si l'image rabelaisienne de la cornucopie ne prévaut pas au sein de la Romancie, les proliférations 'cancéreuses' du langage ne sont toutefois pas absentes du roman: les auteurs, tout en condamnant le foisonnement du roman à épisodes, ne peuvent se défendre l'usage de la digression. Afin de faciliter la tâche du lecteur, ils choisissent de s'adapter aux rythmes de lecture en proposant une présentation matérielle destinée à 'éviter l'ennui d'une lecture suivie', ${ }^{68}$ tout en ménageant le suspens. Outre la parution du roman en volumes séparés, pratique déjà en vigueur chez des auteurs baroques comme La Calprenède ou Mademoiselle de Scudéry, le romancier s'impose d'annoncer rythme et programme de publication, ${ }^{69}$ conférant à ses brochures séparées une dimension plus journalistique. La réception du texte s'en trouve sensiblement modifiée et ouvre la voie au succès du roman-feuilleton du XIXe siècle. Le discours préfaciel représente un parfait objet d'étude pour mettre à jour, dans le discours même du romancier, la double nature de la brièveté - définie comme une qualité ou comme un défaut: $^{70}$

Si elle [ma préface] ennuie le lecteur, je m'en console. L'histoire qui suit lui en paraîtra plus agréable et aura peut-être l'avantage d'avoir su dissiper l'ennui. [...] Si sa brièveté est critiquée, je m'y attends, et je réponds d'avance que c'est la peur d'être froid et languissant qui m'a rendu précis et laconique. ${ }^{71}$

Comme on peut l'observer, le début du XVIIIe siècle est encore fortement marqué par les formes classiques: le caractère naturel du discours préfaciel permet de mesurer la permanence d'anciennes catégories rhétoriques nées des règles de la dispositio. C'est au sein de cette pratique instituée que les romanciers choisissent d'entrer en rupture avec les canons discursifs. L'exemple que nous avons traité - la présence conjointe, à travers la préface, d'un idéal de brièveté et d'une 
fonction informative - laisse apparaitre une tension continuelle, que traduit à un niveau microstructural l'emploi de la prétérition. Frontière du texte par excellence, ${ }^{72}$ la préface résume parfaitement le dilemme poétique qui s'impose aux romanciers du début du siècle. Comme ils l'ont parfaitement compris, la stratégie la plus efficace consiste à subvertir l'assise sur laquelle ils s'appuient. Le maintien des conventions se double d'une stratégie d'autodérision qui pose les fondements du discours métatextuel; ici, respect et subversion des formes sont aussi intimement liés que l'étaient abundantia et brevitas.

\section{STÉPHANIE BOUABANE}

Université de Lille III

\section{Notes}

1 Pierre-François Godart de Beauchamps, Epître (dédicatoire) 'A Madame LCDFB', Les amours d'Ismène et d'Isménias (Paris, 1729) vi.

2 P. C. de Chamblain de Marivaux, 'Préface', La voiture embourbée, 1713-14, CEuvres de jeunesse, éd. F. Deloffre (Paris: Gallimard, 1972) 314-15.

3 Ch. Louis de Secondat, baron de Montesquieu, 'Introduction', Lettres persanes, éd. Jean Starobinski, 1721 (Paris: Gallimard, 1973) 49.

4 Jean-Baptiste de Boyer d'Argens, Lectures amusantes ou les délassements de l'esprit, avec un discours sur les nouvelles (La Haye, 1739) 50.

5 Leven de Templery, La rhétorique françoise, très propre aux gens qui veulent apprendre à parler et écrire avec politesse (Paris, 1698) 94.

6 Cicéron, De l'invention, éd. G. Achard, I, XV, 20 (Paris: Les Belles Lettres, 1994) 75.

7 Ibid.

8 Thomas Laffichard, 'Préface', Le songe de Clidamis, contenant un voyage à Cythère; où l'on trouve plusieurs choses utiles et amusantes (Paris, 1732) n.p.

9 Antoine François Prévost, 'Préface', Le philosophe anglais, ou Histoire de Monsieur Cleveland, fils naturel de Cromwell, écrite par lui-même, et traduite de l'anglais par l'auteur des Mémoires d'un homme de qualité, 1731, CEuvres de Antoine François Prévost, éd. Jean Sgard, II (Grenoble: P.U.G., 1977) 13.

10 'A Ratopolis', Histoire des rats, 1738, 'Discours préliminaire' xiv.

11 Louis Marin, 'Préface', Adélaïde de Witsbury ou La pieuse pensionnaire, avec sa retraite spirituelle de huit jours (Avignon, 1734) iii.

12 Voir la Rhétorique à Alexandre, 1438, a 22.

13 Voir Cicéron, De Oratore et Quintilien, 'Des différents genres de pensées', Institution oratoire, Livre VIII, chap. V: 'Nous désignons par sententiae les traits brillants et surtout à la fin des périodes', 'points lumineux du discours comme les 
yeux de l'éloquence' qui 'frappent l'esprit et ... l'ébranlent souvent d'un seul coup et ... s'y fixent mieux en raison de leur brièveté même et, par le plaisir qu'ils suscitent, ils persuadent.'

14 Ch. de Fieux, Chevalier de Mouhy, 'Préface', Mémoires posthumes du comte de D... $B$..., avant son retour à Dieu, fondé sur l'expérience des vanités humaines (Paris, 1735) vii-viii.

15 Voir Gérard Genette, Seuils (Paris: Le Seuil, 1987) 193.

16 Thomas Laffichard, 'Préface', op. cit.

17 Bertrand Dupriez, 'Prétérition', Gradus. Les procédés littéraires (Paris: U.G.E., '10 / 18 ', 1984) 359. Michel Patillon en donne la définition suivante: 'Dans la prétérition l'orateur feint de laisser de côté quelque chose qu'il n'énonce pas' (Eléments de rhétorique classique [Paris: Nathan, 1990] 89).

18 Gérard Genette, op. cit. 217.

19 Nicolas Lenglet-Dufresnoy, 'Préface', Les princesses malabares, ou Le célibat philosophique. Ouvrage intéressant et curieux, avec des notes historiques et critiques (Andrinople, 1734) n.p.

20 Gérard Genette, op. cit. 216.

21 Madame Meheust, 'Préface', Les mémoires du chevalier de ${ }^{* * *}$ (Paris, 1734) n.p.

22 'Préface' intégrale, La soirée du labyrinthe, débauche d'esprit, suivie du Portefeuille galant. Par Monsieur ${ }^{* * *}$ (Paris, 1732) n.p.

23 G. M. Augustin de Fontanieu, 'Avertissement', La Rosalinde, s.l, 1730, xii.

24 Ibid. vii.

25 Ibid.

26 Ibid. viii.

27 Jean-Baptiste Margat de Tilly, 'Préface', Histoire de Tamerlan, empereur des Mogols et conquérant de l'Asie (Paris, 1739) xvi.

28 Alain-René Lesage, 'Préface du traducteur', Histoire de Guzman d'Alfarache, nouvellement traduite et purgée des moralités superflues, I, 1732 (Paris: Ménard et Desenne, 1825) 4-5.

29 Ibid. 5.

30 Arnauld et Nicole, 'Des enthymèmes et des sentences enthymématiques', $\mathrm{La}$ logique ou l'art de penser, éd. C. Jourdain, 3e partie, chap. XIV (Paris: Gallimard, 1992) 211. Voir également Dominique Bouhours, La manière de bien penser dans les ouvrages d'esprit, éd. Suzanne Guellouz, Livre I, 1705 (Toulouse: Ed. de l'Université de Toulouse-Le Mirail, 1988).

$31 \mathrm{Ibid}$. Voir les passages où Balthasar Gibert reprend les arguments de La logique: il faut ' $n$ 'en dire qu'autant qu'il en faut à l'auditeur pour concevoir le reste par lui-même', car 'l'auditeur a plus de plaisir à suppléer quelque chose de lui-même, qu'à tout entendre de l'orateur. Cela flatte son amour-propre' [La rhétorique ou les règles de l'éloquence (Paris, 1741) 527].

32 Chevalier J. F. J. de Neufville de Brunaubois-Montador, 'Préface', La pudeur, histoire allégorique et morale (Paris, 1739) n.p.

33 Alain-René Lesage, ‘Préface du traducteur', op. cit. 6-7. 
34 Ibid. 11.

35 Claude Prosper Jolyot de Crébillon, 'Extrait d'une lettre de Madame de *** à M de ${ }^{* * \prime}$, Lettres de la marquise de $M^{* * *}$ au comte de $R^{* * *}$, éd. Jean Dagen, 1732 (Paris: Desjonquères, 1990) 47-48.

36 Jean-Baptiste Torchet de Boismêlé, 'Avertissement', Histoire du chevalier du Soleil (Paris, 1740) n.p.

37 La soirée du labyrinthe, débauche d'esprit, suivie du Portefeuille galant (Paris, 1732) 64.

38 Jean-Baptiste de Boyer, marquis d'Argens, op. cit. 24.

39 Je renvoie à ce sujet aux excellents ouvrages de Georges Molinié, Du roman grec au roman baroque. Un art majeur du genre narratif en France sous Louis XIII (Toulouse: Université de Toulouse-Le mirail, 1982) et d'Alain Billault, La création romanesque dans la littérature grecque à l'époque impériale (Paris: P.U.F., 'Ecriture', 1991).

40 Le manque de structure du Polexandre de Gomberville est dénoncé par Madame de Gomez dans La jeune Alcidiane (Paris, 1733) n.p.

41 Henri Pajon, 'Lettre préliminare à Madame la Marquise de ${ }^{* * \prime}$, Histoire $d u$ prince Soly, surnommé Prenany, et de la princesse Feslée (Amsterdam, 1740) iii-iv.

42 Anthony Hamilton, Les quatre Facardins, conte (Paris, 1730) 51.

43 Anthony Hamilton, Le bélier, conte (Paris, 1730) 170.

44 Adrien de La Vieuville d'Orville, Histoire de Lideric premier, comte de Flandres. Nouvelle historique et galante, II (Paris, 1737) 23.

45 Pierre François Guyot Desfontaines, Le nouveau Gulliver, ou Voyage de Jean Gulliver, fils du capitaine Gulliver. Traduit d'un manuscrit anglais, par Monsieur L. D. F. (Paris, 1730) 15, début du chapitre II.

46 Madame Meheust, op. cit. 207.

47 Voir Balthasar Gibert, op. cit. 331: les 'peintures bien faites' sont en effet décrites comme des descriptions 'courtes, vives, naturelles, expressives.'

48 Véritables morceaux de bravoure rhétorique, les progymnasmata étaient des exercices préparatoires qui devaient habituer les élèves à produire tous les types d'énoncés littéraires.

49 Voir par exemple Germain-François Poullain de Saint-Foix, Lettres d'une Turque à Paris, écrites à sa sæur au sérail (Amsterdam, 1730) 94: '(Il manque ici quelque chose, qu'on n'a pu traduire, le manuscrit étant effacé dans cet endroit).'

50 'Préface', Intrigues monastiques, ou L'amour encapuchonné; nouvelles espagnoles, italiennes, françaises (La Haye, 1739) n.p.

51 Henri-Charles de Senneterre, Épitre dédicatoire 'A Madame la marquise de L***', Nouveaux contes de fées (Amsterdam, 1744) ii.

52 Madame de Lintot, 'Préface' (attribuée à Prévost), Trois nouveaux contes des fées, avec une préface qui n'est pas moins sérieuse, (Paris, 1735) xi.

53 Histoire de la vie du marquis d'Ozanne, ou Ses aventures. Ouvrage tiré de ses propres mémoires (Amsterdam, 1740) 270.

54 Madame de Lussan, Anecdotes de la cour de Philippe-Auguste (Paris, 1733) 139.

55 'A Ratopolis' xii.

56 De Lime, L'amour apostat, ou Les aventures de Mr. de ${ }^{* * *}$ et de Mlle ** (Metz, 1739) 6. 
57 P. C. de Chamblain de Marivaux, 'L'école des Lettres', Le paysan parvenu, 1734 (Paris: Le Seuil, 1993) 12. Voir également La vie de Marianne, éd. F. Deloffre, 1ère publication de 1731 à 1745 (Paris: Bordas, 1990) 36, 81 et 221.

58 Ch. de Fieux, Chevalier de Mouhy, 'Préface', Voyages et aventures $d u$ comte de ${ }^{* * *}$ et de son fils (Amsterdam, 1744-45) xi.

59 Louis Marin, 'Préface', op. cit. ix.

60 'A Ratapolis' xii.

61 Ibid.

62 Cicéron, De l'orateur, éd. Edmond Courbaud, II, 326-329, 1928 (Paris: Les Belles Lettres, 1966) 142-43.

63 Ibid.

64 Voir à ce sujet Jean-Baptiste de Boyer d'Argens, op. cit. 65.

65 Georges May, Le dilemme du roman. Etude sur les rapports du roman et de la critique (1715-1761), (Paris: P.U.F., 1963).

66 Voir Mercedes Blanco, Les rhétoriques de la pointe. Baltasar Gracián et le conceptisme en Europe (Genève: Slatkine, tome XXVII, série 3, 1992).

67 Leven de Templery, op. cit. 256.

68 'A Ratopolis' ix.

69 Voir Antoine François Prévost, Le doyen de Killerine, histoire morale composée sur les mémoires d'une illustre famille d'Irlande, et ornée de tout ce qui peut rendre une lecture utile et agréable. Par l'auteur des Mémoires d'un homme de qualité (Paris, 1735) xiv:

'[p]our le Doyen de Killerine, mon dessein est de donner la seconde partie dans six semaines, et de continuer ensuite d'en faire paraître tous les mois. J'ai assez d'avance pour être exact à suivre cet arrangement. Tout l'ouvrage consistera en douze parties, qui composeront à la fin de l'année six volumes.'

70 Voir Etienne Souriau, 'Brièveté', Dictionnaire d'esthétique (Paris: P.U.F., 1990) 271-272: 'Brièveté. Qualité ou défaut de ce qui donne l'impression d'être remarquablement court.'

71 Pierre François Guyot Desfontaines, 'Préface', Histoire de D. Juan de Portugal, fils de D. Pèdre et d'Inès de Castro (Paris, 1724) n.p.

72 Voir Antoine Compagnon, 'Le commencement du livre et la fin de l'écriture', $L a$ seconde main ou le travail de la citation (Paris: Le Seuil, 1979) 341-346 et Gérard Genette, Seuils, op. cit. 